\title{
Immunosuppressive Activity of 13-cis-Retinoic Acid and Prevention of Experimental Autoimmune Encephalomyelitis in Rats
}

\author{
Luca Massacesi, Emanuela Castigli, Marco Vergelli, Jacopo Olivotto, Anna Laura Abbamondi,* \\ Francesco Sarlo, and Luigi Amaducci \\ Dipartimento di Scienze Neurologiche e Psichiatriche Universitd degli Studi di Firenze, 50134 Florence, Italy; \\ *Istituto di Neurologia, Università Cattolica, Policlinico Gemelli, 00100 Rome, Italy
}

\begin{abstract}
Some activities of retinoids on cellular and humoral immunity have been described, but the available data are conflicting or obtained at concentrations that are toxic in vivo. In this study, we demonstrate that 13-cis-retinoic acid (13-cRA), a retinoid well tolerated in human therapy, can suppress $T$ cell-mediated immunity in rats. Treatment with pharmacological concentrations of 13-cRA prevented active as well as passive transfer experimental autoimmune encephalomyelitis (EAE) and suppressed lymphocyte responsiveness to $T$ cell mitogens, suggesting that the drug activity included suppression of an effector $T$ cell response. In addition, mitogen- and antigen-induced lymphocyte proliferation was inhibited in vitro in the presence of concentrations of 13-cRA equivalent to or less than those achieved in vivo, further suggesting that the prevention of EAE was due to a suppressive activity on $T$ cell-mediated immunity. The immunosuppressive activity of 13-cRA included suppression of interleukin 2, whose production was inhibited in splenocytes. These data indicate that, in an in vivo mammalian system, 13-cRA exerts a suppressive activity on $T$ cell-mediated immunity intensive enough to suppress an ongoing immune response, and that this effect can be achieved at nontoxic concentrations that may also be attained in human therapy. (J. Clin. Invest. 1991. 88:1331-1337.) Key words: experimental autoimmune encephalomyelitis • central nervous system • isotretinoin • $T$ cell-mediated immunity $\bullet$ immunotherapy
\end{abstract}

\section{Introduction}

Retinoids are the class of natural and synthetic derivatives of vitamin A. The best known molecule of this class, all-transretinoic acid (RA), ${ }^{1}$ plays a physiological role in morphogenesis

A preliminary report of one experiment covered in this article was published as an extended abstract in the proceedings of the 1989 meeting of European Committee for Treatment in Multiple Sclerosis.

Address reprint requests to Dr. Massacesi, Dipartimento di Scienze Neurologiche e Psichiatriche, Viale Morgagni 85, 50134 Firenze, Italy.

Received for publication 5 February 1991 and in revised form 30 April 1991.

1. Abbreviations used in this paper: CFA, complete Freund's adjuvant; EAE, experimental autoimmune encephalomyelitis; IL-2sup, interleukin 2 supernatant(s); MBP, myelin basic protein; p.i., postimmunization; RA, all-trans-retinoic acid; 13-cRA, 13-cis-retinoic acid; RAR, $\mathrm{RA}$ receptor; $\mathrm{SC}$, spleen cell; $\mathrm{SI}$, stimulation index.

J. Clin. Invest.

(c) The American Society for Clinical Investigation, Inc.

$0021-9738 / 91 / 10 / 1331 / 07 \quad \$ 2.00$

Volume 88, October 1991, 1331-1337 and cell differentiation. Its 13-cis-isomer (13-cis-retinoic acid, 13-cRA), which is better tolerated for systemic administration, is also used in the therapy of various dermatologic and neoplastic diseases (1-3).

RA (tretinoin), 13-cRA (isotretinoin), and other retinoids have been described to exert a number of suppressive activities on $\mathrm{T}$ cell functions, including a decrease in production of interleukins (IL, 4-15). However, immunostimulatory effects of 13cRA and other retinoids on cellular and humoral immunity have also been reported (16-24). These observations indicate that the interactions of these molecules with the immune system are complex and not completely clarified. Nonetheless, the established and potential applications of retinoids in human therapy demand deeper investigation of their effects on the immune system, particularly at pharmacological concentrations.

In a previous article we reported that experimental autoimmune encephalomyelitis (EAE) was suppressed by RA (25). $\mathrm{EAE}$ is an autoimmune disease of the central nervous system (CNS) inducible in susceptible animals by immunization with myelin antigens or by passive transfer of sensitized $T$ cells to syngenic recipients (26-34). EAE induced in Lewis rats has been used as a generic in vivo model of autoimmune disorders, because of the ease of detecting and quantitating disease activity and the consistency with which this rat strain develops a $T$ cell-mediated delayed-type hypersensitivity-like autoimmune response $(26,27)$. In rodents, EAE is mediated by $\mathrm{CD}^{+}, \mathrm{CD}^{-}$, and IL-2 receptor-positive T lymphocytes which induce perivascular inflammatory infiltrates in CNS by the end of the 2nd wk postimmunization (p.i.), in accordance with the onset of neurological signs, whereas encephalitogenic $T$ lymphocytes can be recovered from peripheral lymphoid organs or peripheral blood, beginning 1 wk p.i. (30-34). In passive transfer EAE, CNS inflammatory infiltrates and neurological signs are present 5 or $6 \mathrm{~d}$ post-transfer of encephalitogenic $\mathrm{T}$ lymphocytes (30-34).

The suppression of EAE by RA indicates a possible suppressive activity of this drug on immune-mediated diseases, but its usefulness was limited by its toxicity, and therefore its possible immunosuppressive activity in the suppression of EAE was not studied further (25). However, 13-cRA, which has been demonstrated to retain most of the pharmacological activities of RA, is better tolerated for in vivo administration (1-3). This finding suggests that 13-cRA could represent an optimal retinoid for experimentation in autoimmune diseases, but the activity of pharmacological doses of this drug on $\mathrm{T}$ cell-mediated immunity has not been directly investigated (4).

For these reasons, we studied the in vivo effects of nontoxic pharmacological concentrations of 13-cRA in EAE, while the activity of the drug on T cell-mediated immunity was studied in splenocytes in vitro. We report that 13-cRA prevented EAE 
and suppressed $T$ cell response during the efferent phase of the immune response. The immunosuppression was functional and reversible, and was obtained at concentrations that can be achieved in human therapy, without inducing major side effects.

\section{Methods}

\section{Preparation of the antigens}

Myelin and myelin basic protein (MBP) were purified from guinea pig spinal cords (Pel Freeze Biologicals, Rogers, AR) according to the method of Norton and Poduslo (35) and Deibler et al. (36), respectively. The preparations were lyophilized, and stored at $-20 \mathrm{C}$.

\section{Induction of $E A E$}

Female Lewis rats (175-225 g; Charles River Breeding Laboratories, Inc., Wilmington, MA) were immunized with an emulsion of either $(a)$ $1 \mathrm{mg}$ of guinea pig myelin, dispersed in $0.5 \mathrm{ml}$ of complete Freund's adjuvant (CFA) containing $3 \mathrm{mg} / \mathrm{ml}$ of Mycobacterium tuberculosis H37RA or (b) $50 \mu \mathrm{g}$ of guinea pig MBP in $0.1 \mathrm{ml}$ of CFA. The animals were injected intradermally in both hind foot pads. As "normal" control, six additional rats were sham-immunized with $0.5 \mathrm{ml}$ of CFA only.

\section{Assessment of EAE}

Two experiments were designed in order to follow the clinical signs, and to describe laboratory parameters at day 12 p.i. In experiment 1 , animals were assessed daily for weight and clinical signs for 3 wk. Clinical disease severity was graded on a scale of $1-5$ as follows: 0 , no signs; 1, tail paralysis; 2, tail paralysis and hind leg weakness; 3 , hind leg paralysis; 4 , tetraplegia; 5 , death. In experiment 2 , the animals were killed at day 12 p.i. in order to compare laboratory parameters and clinical signs. Spinal cord and brain were examined for the presence of perivascular cuffs. The intensity of the perivascular infiltrates was arbitrarily graded from 1 to 3 by two independent observers. Total DNA concentration was assayed in the spinal cord, according to Smith et al. (37), as a parameter of inflammatory cell infiltrates. At the time of sacrifice, peripheral blood samples were collected. Absolute and relative lymphocyte counts were carried out using an hemocytometer and Giemsa stained blood smears.

\section{Spleen cell (SC) response to Con A and antigens}

Spleens were collected at day 10 p.i. and passed through a stainless steel screen mesh. The single cell suspensions were separated from erythrocytes in a Ficoll-Hypaque density gradient centrifugation and washed in HBSS (Flow), resuspended in a final concentration of $2 \times 10^{6} / \mathrm{ml}$ of RPMI 1640 (Flow) supplemented with 5\% heat-inactivated FCS (Flow Laboratories, Inc., McLean, VA), 1\% L-glutamine, $1 \%$ Na-pyruvate (Flow Laboratories, Inc.), 1.25\% Hepes buffer (Flow Laboratories, Inc.), $1 \%$ nonessential amino acids, $1 \%$ penicillin-streptomycin (Flow Laboratories, Inc.), and $5 \times 10^{-5} \mathrm{M} 2$-mercapoethanol (2-ME). Proliferative assays in response to $6.25 \mu \mathrm{g} / \mathrm{ml}$ myelin and $50 \mu \mathrm{g} / \mathrm{ml} \mathrm{MBP}$ and to $4 \mu \mathrm{g} / \mathrm{ml}$ Con A, were carried out in 96-microwell plates using $2 \times 10^{5}$ cells per well. $\left[{ }^{3} \mathrm{H}\right]$ thymidine incorporation was measured by scintillation counting and the magnitude of the proliferative respose was expressed as mean counts per minute (cpm) or as stimulation indexes (SI; stimulated culture cpm/unstimulated culture cpm).

\section{Preparation of IL-2-containing supernatant (IL-2sup)}

SC were removed from Lewis rats and cultured at $5 \times 10^{6} / \mathrm{ml}$ in presence of $4 \mu \mathrm{g} / \mathrm{ml}$ Con A as previously described (38). After $72 \mathrm{~h}$ the IL-2sup were removed, treated with $20 \mathrm{mg} / \mathrm{ml} \alpha$-methyl-mannoside, and frozen.

\section{$I L-2$ assay}

The IL-2 activity of the IL-2sup was assayed using the murine IL-2-dependent cell line, CTLL-2 (American Type Culture Collection, Rock- ville, MD) as previously described (39). Briefly $10^{4}$ cells per well were added to $200 \mu \mathrm{l}$ of culture medium containing increasing dilutions of IL-2sup. $48 \mathrm{~h}$ later the cultures were pulsed with $\left[{ }^{3} \mathrm{H}\right]$ thymidine for an additional $18 \mathrm{~h}$. Data from two separate experiments were fitted using a program for analysis of dose-response curves (40). A possible CTLL-2 response to IL-4 was controlled in a separate assay and excluded: up to $50 \mathrm{U} / \mathrm{ml}$ of mouse IL-4 did not elicit CTLL-2 proliferation.

\section{Passive transfer of EAE}

Sensitized SC, harvested at day 10 from untreated immunized rats, were cultured in 24-well plates at a concentration of $5 \times 10^{6} / \mathrm{ml}$ with 4 $\mu \mathrm{g} / \mathrm{ml}$ Con A for $3 \mathrm{~d}$ as previously described (41). After $72 \mathrm{~h}$ of culture, $4 \times 10^{7}$ viable SC per animal were injected i.p. to syngeneic naive recipients.

\section{3-cRA}

13-cRA was kindly provided by Dr. W. Bollag (Hoffmann-LaRoche, Basel). The powder was aliquoted in oxygen-free vials and stored at $4^{\circ} \mathrm{C}$ in the dark. Any procedure utilizing 13-cRA was carried out in dim light.

In vivo studies. On each day of treatment, the 13-cRA was suspended in corn oil and administered by gastric intubation, in two daily doses; matched controls received the vehicle only. In active EAE the animals were treated from days 6 to 11 p.i., whereas in passive EAE, from days 1 to 6 after the cell transfer. 13-cRA was suspended in corn oil at a concentration of $7.5 \mathrm{mg} / \mathrm{ml}$. Each rat received $1 \mathrm{ml}$ of suspension per $200 \mathrm{~g}$ of weight twice a day, in order to administer a total of 75 $\mathrm{mg} / \mathrm{kg}$ per d. At day 5 of treatment (day 10 p.i.), sera from $37 \mathrm{immu-}$ nized rats were collected at hours $1,2,4,8$, and 12 after the morning administration of the drug. To study the pharmacokinetics, 13-cRA concentrations were assayed in the serum samples by HPLC as previously described $(42,43)$, and the average serum concentration over 24 $\mathrm{h}$ was determined.

In vitro studies. 13-cRA was dissolved at different concentrations in dimethyl sulfoxide (DMSO); the stock solutions were then diluted in the culture medium at $0.01 \%$ DMSO final concentration. Control cultures were exposed to $0.01 \%$ DMSO only.

\section{Results}

In vivo studies. In control rats, the onset of EAE signs was observed at day $11 \pm 1$ p.i. (Fig. 1, Table I), whereas proliferative response to the $\mathrm{Ag}(\mathrm{SI}=4.5)$, was observed in $\mathrm{SC}$ beginning at day 6 (data not shown). In order to investigate the activity of 13-cRA on the effector mechanisms of the immune response in EAE, the animals immunized at day 0 with myelin were therefore treated from days 6 to 11 (Fig. 1, Table I), whereas the animals receiving cell transfer at day 0 were treated from days 1 to 6 (Table II). At day 5 of treatment, $75 \mathrm{mg} / \mathrm{kg}$ per d 13-cRA induced an average serum concentration during $24 \mathrm{~h}$ of $5.4 \pm 5.2 \times 10^{-6} \mathrm{M}$.

No major side effects were associated with 13-cRA treatment. In some treated animals a modest degree of weight loss (always $<10 \%$ ) was observed (data not shown). No diarrhea or other signs of gastrointestinal irritation were noted.

As shown in Fig. 1 a delayed and less severe course of the disease was observed in animals receiving 13-cRA. Analysis at day 12 of the frequency of neurological signs and of the presence of inflammatory infiltrates in spinal cords showed that the disease was significantly suppressed ( $\chi^{2}$ test: $P<0.001$; Table I). The onset of neurological signs, observed a few days after the suspension of the treatment, indicated that discontinuation of the therapy resulted in partial loss of the protective effects (FIg. 1). The highly significant suppression of DNA concentration in the spinal cords of the treated animals quantitatively con- 


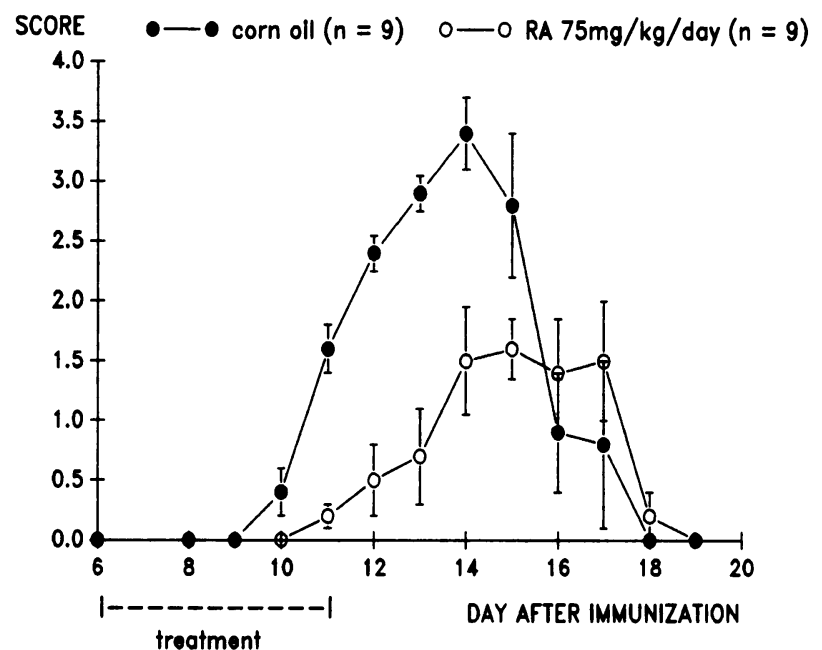

Figure 1. Effect of 13-cRA on the course of neurological signs in EAE. 13-cRA and corn oil were administered from days 6 to 11 after immunization for induction of EAE. Each point represents the mean of nine observations \pm SE. A delayed and less severe course of EAE is shown by the group receiving 13-cRA. The symptom score is reported in the text.

firmed the suppression of the inflammatory response in the CNS (Table I). The characteristic lymphopenia described in rats during the course of EAE and during endogenous corticosteroid release (44-46) was observed in the vehicle-, but not in the 13-cRA-treated animals (Table I). In order to establish if the suppression of EAE occurred in presence of in vivo effects of the treatment on $\mathrm{T}$ cell functions, lymphocyte response to Con A was studied at day 10, immediately before the onset of the disease. At $8 \mathrm{~h}$ after the oral administration, when the 13cRA serum concentration was $2.8 \pm 2.4 \times 10^{-6} \mathrm{M}$, a lower proliferative response of lymphocytes from the 13-cRA-treated animals was observed (two-tailed Mann-Whitney $U$ test, $2 P$ $=0.02$; Fig. 2).

Table I. Effects of 13-cRA in Active EAE at Day 12

\begin{tabular}{lcccc}
\hline & & \multicolumn{2}{c}{ Inflammatory infiltrates } & \\
\cline { 3 - 4 } Treatment $^{*}$ & Neurological signs $^{*}$ & $\begin{array}{c}\text { Perivascular } \\
\text { cuffs }^{*}\end{array}$ & DNA $^{*}$ & $\begin{array}{c}\text { Lymphocyte } \\
\text { counts }\end{array}$ \\
\hline & & & $\mu g / m g$ & \\
13-cRA & $8 / 26(0.8 \pm 0.1)$ & $12 / 26(0.7)$ & $6.0 \pm 0.5$ & $103 \pm 17$ \\
& $P<0.001$ & $P<0.001$ & $P<0.001$ & $P<0.001$ \\
Oil & $25 / 26(2.2 \pm 0.1)$ & $26 / 26(1.7)$ & $9.6 \pm 0.1$ & $53 \pm 7$ \\
& & & & \\
\hline
\end{tabular}

* $75 \mathrm{mg} / \mathrm{kg}$ per d 13-cRA in corn oil or corn oil only from days 6 to 11 p.i.

₹ The numbers represent the frequency in each group, of animals with neurological signs or perivascular cuffs in the spinal cord. In parenthesis are reported the mean scores of the neurological signs or of the intensity of the inflammatory infiltrates ( $\chi^{2}$ test).

'Spinal cord mean DNA concentrations expressed as $\mu \mathrm{g}$ DNA/mg of protein \pm 2 SE. The mean DNA concentration in six sham-immunized animals was 5.6 \pm 0.2 . (Student's $t$ test).

" Percentage \pm 2 SE of the values observed in sham-immunized animals (mean \pm 2 SE of six observations $=4,416+385$ ). (MannWhitney test).
Table II. Effects of 13-cRA on EAE Passive Transfer*

\begin{tabular}{lccc}
\hline \multicolumn{2}{c}{ Treatment } & & \\
\hline In vitro" & In vivo" & Neurological signs & Perivascular cuffs $^{*}$ \\
\hline DMSO & Oil & $16 / 18$ & $18 / 18$ \\
13-cRA & Oil & $0 / 12$ & $0 / 12$ \\
DMSO & 13-cRA & $0 / 12$ & $0 / 12$ \\
& & & \\
\hline
\end{tabular}

* $40 \times 10^{6} \mathrm{SC}$ were injected i.p. in syngenic recipients.

₹ The numbers represent the frequency in each group, of animals with neurological signs or perivascular cuffs in the spinal cord.

Before transfer, the SC were cultured for $72 \mathrm{~h}$ with Con A, in presence of $0.01 \%$ DMSO or $10^{-6} \mathrm{M} 13-\mathrm{cRA}$ dissolved in $0.01 \%$ DMSO.

"From days 1 to 6 after cell transfer, $75 \mathrm{mg} / \mathrm{kg}$ per d of 13-cRA was administered in $1 \mathrm{ml}$ of corn oil. Control animals received corn oil only.

The in vivo activity of 13-cRA on the efferent limb of the immune response was also studied in passive transfer EAE. Animals treated with the vehicle developed neurological signs from days $6 \pm 1$ to $9 \pm 1$ after transfer, and perivascular cuffs were observed in spinal cords at day 11 (Table II). By contrast, animals receiving 13-cRA, from days 1 to 6 after cell transfer, developed neither neurological signs nor perivascular infiltrates in the spinal cord (Table II).

In vitro studies. In order to assess whether the suppression of EAE was due to a direct activity of the drug on T cell-mediated immunity, we studied in vitro the effects of 13-cRA on $S C$ collected from untreated animals immunized with myelin or MBP. As shown in Table III, exposure of SC to 13-cRA concentrations equivalent to or less than those observed in vivo suppressed lymphocyte proliferation to antigens and to Con $\mathrm{A}$ in a dose-dependent manner. It is noteworthy that the background proliferations were not affected by the same concentrations of 13-cRA, indicating that up to $96 \mathrm{~h}$ in vitro exposure to the drug did not exert direct toxicity in lymphocytes. Moreover, exposure of the SC to $10^{-6} \mathrm{M} 13-\mathrm{cRA}$ during in vitro activation with Con $\mathrm{A}$, before passive transfer, abrogated the

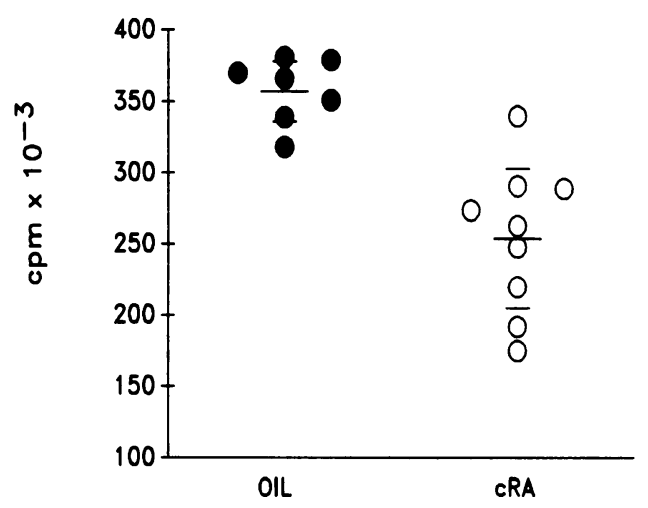

Figure 2. In vivo immunosuppressive activity of 13-cRA during the development of EAE. Each point represents quadruplicate proliferations of SC collected at day 5 of treatment (day 10 p.i.) and cultured for $48 \mathrm{~h}$ in presence of ConA (SE of $<15 \% \mathrm{cpm}$ of the mean). When the serum concentration of the drug was $2.8 \pm 2.4 \times 10^{-6} \mathrm{M}$, animals receiving $13-c R A(0)$ showed lower lymphocyte proliferation than the oil treated controls $(\bullet)$; mean \pm SE: $13-c R A=252 \pm 47$; OIL $=355 \pm 20$; Mann-Whitney U test: $P=0.02$ ). 


\begin{tabular}{|c|c|c|c|c|c|}
\hline & & DMSO* & RA $10^{-8} \mathrm{M}$ & RA $10^{-7} \mathrm{M}$ & RA $10^{-6} \mathrm{M}$ \\
\hline \multicolumn{6}{|c|}{$c p m$} \\
\hline & Medium* & $4,420 \pm 567^{8}$ & $5,140 \pm 943$ & $2,920 \pm 348$ & $6,740 \pm 1,130$ \\
\hline \multirow[t]{3}{*}{ Exp. 1} & Con $\mathbf{A}$ & $319,000 \pm 13,400(72.2)$ & $114,000 \pm 9,140(22.3)$ & $95,500 \pm 15,000(32.7)$ & $70,600 \pm 5,870(10.5)$ \\
\hline & Myelin $(6.25 \mu \mathrm{g} / \mathrm{ml})$ & $21,000 \pm 3,940(4.8)$ & $11,400 \pm 2,530(2.2)$ & $10,200 \pm 1,790(3.5)$ & $11,500 \pm 712(1.7)$ \\
\hline & Medium & $11,600 \pm 3,010$ & $9,630 \pm 565$ & $14,700 \pm 1,290$ & $5,490 \pm 1,030$ \\
\hline \multirow[t]{3}{*}{ Exp. 2} & Con A & $308,000 \pm 28,400(26.4)$ & $228,000 \pm 14,700(23.7)$ & $191,000 \pm 19,500(12.9)$ & ND \\
\hline & Myelin $(6.25 \mu \mathrm{g} / \mathrm{ml})$ & $41,400 \pm 7,720(3.5)$ & $22,400 \pm 4,170(2.3)$ & $19,400 \pm 3,020(1.3)$ & $15,000 \pm 2,180(2.73)$ \\
\hline & Medium & $8,450 \pm 2,030$ & $6,390 \pm 1,520$ & $6,650 \pm 1,460$ & $7,660 \pm 1,390$ \\
\hline \multirow[t]{3}{*}{ Exp. 3} & Con A & $169,000 \pm 6,270(20.0)$ & $93,000 \pm 7,110(14.5)$ & $86,800 \pm 6,870(13.1)$ & $66,500 \pm 9,520(8.7)$ \\
\hline & $\mathrm{MBP}(50 \mu \mathrm{g} / \mathrm{ml})$ & $37,800 \pm 4,520(4.5)$ & $22,400 \pm 3,070(3.5)$ & $23,100 \pm 4,790(3.5)$ & $15,000 \pm 2,310(1.9)$ \\
\hline & Medium & $2,540 \pm 422$ & $3,200 \pm 545$ & $2,610 \pm 278$ & $2,650 \pm 4,170$ \\
\hline \multirow[t]{2}{*}{ Exp. 4} & Con A & $139,000 \pm 10,200(54.6)$ & $68,700 \pm 3,700(21.4)$ & $59,000 \pm 10,000(22.6)$ & $50,300 \pm 3,460(18.9)$ \\
\hline & $\mathrm{MBP}(50 \mu \mathrm{g} / \mathrm{ml})$ & $14,800 \pm 2,000(5.8)$ & $2,830 \pm 411(0.9)$ & $3,410 \pm 483(1.3)$ & $2,810 \pm 292(1.1)$ \\
\hline
\end{tabular}

* Myelin, MBP, and Con A were dissolved in the culture medium at $6.25,50$, and $4 \mu \mathrm{g} / \mathrm{ml}$, respectively. ${ }^{\ddagger}$ Each culture contained $0.01 \%$ DMSO. The values express $\mathrm{cpm} \pm \mathrm{SE}$ of quadruplicate cultures. Stimulation indices (SI) are reported in parenthesis. The cells were collected from rats immunized with myelin in experiments 1 and 2, and with MBP in experiments 3 and 4.

capacity of encephalitogenic lymphocytes to transfer EAE (Table II).

The previous experiments, indicating that $\mathrm{T}$ cell-mediated immunity is at least one of the targets of the immunological activity of 13-cRA, led us to study the effect of 13-cRA on lymphokine production by T cells. As expected, IL-2sup prepared in the presence of $10^{-6} \mathrm{M}$ 13-cRA showed a lower IL-2 activity, expressed as the proliferation of the IL-2-dependent $T$ cell line CTLL-2, compared to control IL-2sup $(P=0.048$, Fig. $3)$. However, the proliferation of the CTLL-2 induced by optimal dilutions of IL-2sup was not inhibited by the addition of $10^{-6} \mathrm{M}$ 13-cRA (data not shown). Suppression of IL-2 produc-

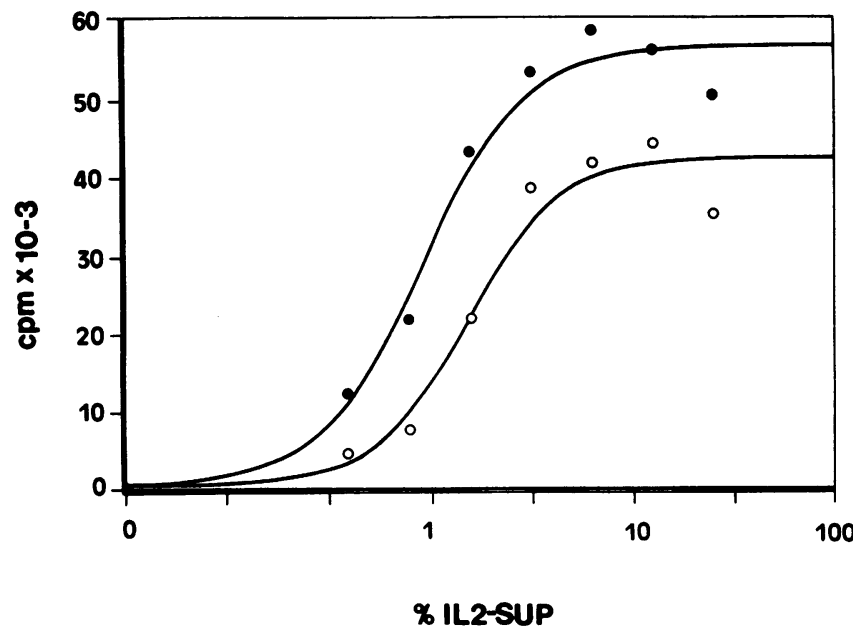

Figure 3. Suppression of IL-2 production in presence of $10^{-6} \mathrm{M} 13-$ cRA. The curves represent the response of the IL-2-dependent $T$ cell line CTLL-2 to percent dilutions of IL-2 sup produced by SC in presence of $10^{-6} \mathrm{M} 13-\mathrm{cRA}$ (RA IL-2sup, O), and to control IL-2sup (๑). The dilution of RA IL-2sup exerting 50\% of the maximal activity $\left(E D_{s 0}\right)$ was significantly higher $($ mean $\pm \mathrm{SE}=1.65 \% \pm 0.21)$ than the control ED $\mathrm{ED}_{50}(0.88 \% \pm 0.11 ; P=0.048)$. tion, but not of IL-2 activity, is therefore one effect of 13-cRA on $T$ cell-mediated immunity.

Finally, the effects of different times of exposure to $10^{-6} \mathrm{M}$ 13-cRA were examined in proliferating $T$ lymphocytes during a standard 96-h stimulation assay in presence of Con A. 13cRA reduced a SC proliferative response to the mitogen in a time-dependent manner, if it was added during the first $48 \mathrm{~h}$ of the assay, but did not show any effect if the Con A-stimulated $\mathrm{SC}$ were exposed to 13-cRA during the second $48 \mathrm{~h}$ (Fig. 4). This experiment demonstrates that exposure to $10^{-6} \mathrm{M} 13-$ cRA for up to $48 \mathrm{~h}$ does not affect viability of stimulated proliferating lymphocytes. Because under these experimental conditions at least one mitotic cycle is completed in $\mathbf{4 8} \mathrm{h}$, thymidine incorporation equivalent to $100 \%$ of the untreated cultures, indicate absence of antimitotic activity of the drug.

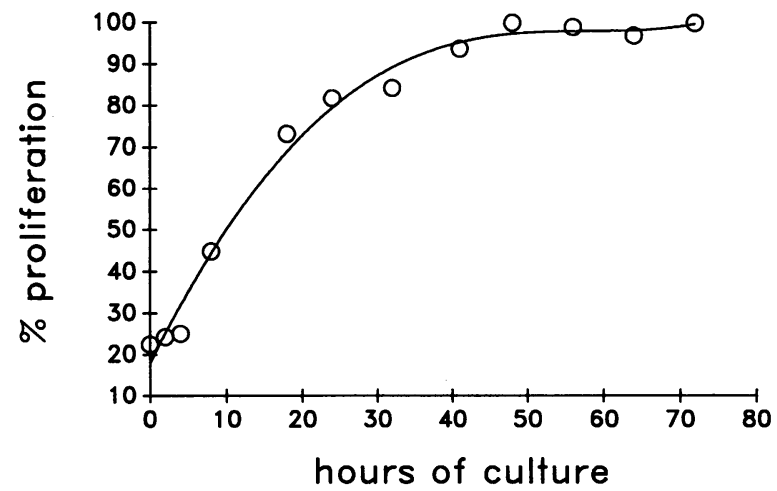

Figure 4. Effects of different times of exposure of SC to $10^{-6} \mathrm{M} 13-$ cRA during a standard proliferation assay with Con A. Triplicate 96-h cultures were exposed to 13-cRA starting from the hour indicated on abscissa: each point represents the percentage proliferation of the 13-cRA exposed cultures in respect to control cultures not exposed to 13-cRA. ( $\Delta$-cpm of 13-cRA exposed cultures/ $\Delta$-cpm of DMSO-exposed cultures $\times 100$ ). 13-cRA suppresses the response to the mitogen in a time-dependent manner if added during the first part of the assay, but has no effect if added during the last $48 \mathrm{~h}$. 


\section{Discussion}

We have studied the effects of 13-cRA on in vivo and in vitro models of cellular immunity, at concentrations that are well tolerated in human therapy $(1-3,47-50)$. 13-cRA administration during the development of the efferent limb of the immune response significantly suppressed the clinical and histopathological signs of active EAE. Suppression was almost complete as long as the animals received 13-cRA, whereas discontinuation of the treatment resulted in a loss of the protective effect. However, passive transfer EAE was completely prevented and discontinuation of 13-cRA $6 \mathrm{~d}$ after passive transfer resulted in no late disease.

In these experiments, suppression of EAE was achieved using a treatment protocol that was not associated with major side effects and that induced serum concentrations equivalent to the highest reported to be tolerated in human therapy. Administration of drug boluses through gastric intubation permitted a precise control of the dosages, but did not allow an optimal absorption of the drug. For this reason and because of the high turnover rate of 13-cRA described in rodents (1-3), high oral dosages were required to achieve the desired serum concentrations. Preliminary experiments indicated that diarrhea and other signs of gastrointestinal intolerance (e.g., accelerated weight loss) developed in some animals by day 7 of 13-cRA therapy. For this reason a 6-d treatment regimen was selected. In humans, equivalent serum concentrations can be reached using much lower dosages of 13-cRA and such regimens are well tolerated even during chronic administration (47-50).

The response of SC to the T cell mitogen Con A was also inhibited during the treatment, indicating that the suppression of the disease occurred in presence of in vivo inhibition of $T$ cell functions. The possibility that this inhibition was not functional but resulted from a direct lymphocytotoxic activity of the drug was excluded by the observation that, at day 7 from the beginning of the treatment, animals receiving 13-cRA had the same number of circulating lymphocytes as the "normal" controls which were sham-immunized with CFA only. The possibility that subclinical toxicity of 13-cRA may have induced release of endogenous corticosteroids was investigated using peripheral blood lymphocyte count as a marker: indeed, in rats exposed to stress, high levels of corticosteroids are secreted and a corresponding lymphopenia is usually observed $(44-46,51)$. In the present experiments, the rats treated with 13-cRA showed lymphocyte counts equivalent to the sham-immunized rats injected only with CFA, whereas the animals receiving corn oil showed the usual lymphopenia described in EAE (Table I, reference 46). This observation indicates that the 13-cRA administration did not induce relevant endogenous corticosteroid release, and therefore that the suppression of the disease was directly due to the treatment. The immunosuppressive activity of 13-cRA was also observed in vitro. Continuous exposure to 13-cRA concentrations equivalent to or less than those observed in vivo during EAE, suppressed Con A- and Ag-induced lymphocyte proliferation in a dose- and time-dependent manner, with maximal activity at $10^{-6} \mathrm{M}$ (Table III), which decreased as a function of time when added after the lymphocyte activation (Fig. 4). This concentration prevented passive transfer of EAE by encephalytogenic lymphocytes (Table II) and reduced IL-2 production (Fig. 3) without lymphocytotoxic effect in vivo (Table I) as well as in vitro (Fig. 4). Taken altogether, the data reported in this paper indicate that pharmaco- logical concentrations of 13-cRA are immunosuppressive and can prevent an ongoing $\mathrm{T}$ cell-mediated autoimmune disease even when administered exclusively during the development of the efferent limb of the immune response. An immunosuppressive activity intensive enough to control an ongoing immune response is shown also by cyclosporine $\mathrm{A}$ but not by cytotoxic drugs $(52,53)$. These drugs can suppress EAE if given prophylactically, but not if administered when the immune system has already mounted an effector response (53). It is likely that 13cRA treatment does not specifically delete clones of Ag-responsive $\mathrm{T}$ cells, and the immunosuppressive activity is probably due to a nonspecific effect on one or more subpopulations of immunocompetent cells, which include suppression of IL-2 production. Indeed, the described activities of 13-cRA on T cell-mediated immunity and IL-2 production were observed stimulating an unselected SC population which included accessory cells such as macrophages and dendritic cells. In addition, suppressive activities of RA on accessory cell functions has been previously described $(14,54)$, suggesting that 13-cRA activity on IL-2 production as well as on $\mathrm{T}$ cell proliferation, could in part be indirect and mediated by an effect on accessory cells. The suppression of the $T$ cell proliferative response as well as of IL-2 production, could be therefore due to a direct effect of 13-cRA on T-lymphocytes or could be indirect and mediated by other soluble factors produced by a number of immunocompetent cells. It is noteworthy, for example, that RA stimulates the production of transforming growth factor- $\beta$, one of the most powerful immunosuppressive cytokines, which is produced by many immunocompetent and nonimmunocompetent cells $(55,56)$. Nonetheless, the possibility of a direct effect of 13-cRA on IL-2 production by T lymphocytes is indirectly suggested by the reported inhibition by RA of diacylglycerol induced ornithine-decarboxylase activity, an early event that plays a key role in T lymphocyte activation (5-7). In addition, the described supression of IL-2 production is consistent with the recent observation that another experimental retinoid, Ro 15-0778 (temarotene, Hoffmann-LaRoche, Basel), inhibits phytohemagglutinin-induced production of IL-2 and IFNgamma in human T-lymphocytes (W. Bollag, personal communication). Temarotene has also been reported to exert a marked therapeutic activity on lichen planus, a human $\mathrm{T}$ cell-mediated dermatological disease (57). The possibility that 13-cRA acts on multiple targets of the immune activity must therefore be taken into account when results obtained under different experimental conditions are compared and may at least in part explain the large number of conflicting results reported in the literature (for review, see reference 4).

The similar pharmacological activities of RA and 13-cRA and the existence of an intracellular retinoic acid binding protein that binds both these retinoids (58) suggest that the two isomers could act through a common receptor. Five different RA receptors (RARs), each one encompassing multiple isoforms, have been so far described (59-67). At least three of these receptors bind RA as well as other natural and synthetic retinoids (59-62), including 13-cRA (63), although with less affinity. In addition, some degree of cis-trans isomerization to RA may in part contribute to the activity of 13-cRA. The RARs are differentially expressed in various tissues and exhibit different affinities for RA and other retinoids $(59,64)$, suggesting that they may have distinct roles in mediating a number of different activities of these molecules. Very few genes activated by the RARs are known so far $(61,64,65)$, but it is noteworthy 
that these receptors are highly conserved from rodents to humans and are expressed in both human and rat spleen, and that the optimal RA concentration inducing maximal transcription by the RARs is $10^{-6} \mathrm{M}(59,60,63-66)$, the same nontoxic concentration that we have shown to be active on $\mathrm{T}$ cell-mediated immunity. In addition, RARs are part of a superfamily of nuclear receptors that also mediate the activity of steroid and thyroid hormones (67), supporting the hypothesis that RA functions in a fashion analogous to these hormones. Moreover, it must be emphasized that steroid and thyroid hormones can repress gene expression as well as activate it (67). It is possible therefore to hypothesize that, like glucocorticoids, 13-cRA exerts suppressive activity on $\mathrm{T}$ cell-mediated immunity through a receptor whose expression varies in different cell populations and under different experimental conditions. Nonetheless, the observations presented in this article establish that, in an in vivo mammalian system, the net result of 13-cRA activity is a suppressive effect on $\mathrm{T}$ cell-mediated immune response, and that this activity is intensive enough to suppress an ongoing immune response at nontoxic concentrations which may also be reached in human therapy. Considering that the serum concentrations of 13-cRA induced in this study can be chronically maintained in human therapy with minor side effects (1-3, 47-50), the experimental use of 13-cRA also in human chronic $T$ cell-mediated autoimmune diseases can now be proposed. In addition, the prevention of EAE, a model of human T cellmediated disease of the CNS (27), and the excellent bioavailability in the CNS of retinoids $(68,69)$, suggest that multiple sclerosis patients could constitute an appropriate population for a phase 1 clinical trial.

\section{Acknowledgments}

The authors wish to thank Dr. Laura Raimondi for the HPLC assay of 13-cRA and Dr. Dale McFarlin and Dr. Richard McCarron for helpful discussion. The authors are also grateful to Dr. Michael Sporn for help in the revision of the manuscript.

This study was supported by a grant of the Italian National Research Council. Dr. Massacesi was a recipient of a fellowship of the Italian National Research Council.

\section{References}

1. Bollag, W. 1983. Vitamin A and retinoids: from nutrition to pharmacotherapy in dermatology and oncology. Lancet. i:860-863.

2. Goodman, D. S. 1984. Vitamin A and retinoids in health and disease. $N$ Engl. J. Med. 310:1023-1031.

3. Sporn, M. B., A. B. Roberts, and D. S. Goodman. 1984. The Retinoids Academic Press, Inc., New York.

4. Shapiro, P. E., and R. L. Edelson. 1985. Effect of retinoids on the immune system. In Retinoids: New Trends in Research and Therapy. R. Saurat, editor. S. Karger, Basel. 225-235.

5. Kensler, T. W., and G. C. Mueller. 1978. Retinoic acid inhibition of the comitogenic action of mezerein and phorbol esters in bovine lymphocytes. Cancer Res. 38:771-775.

6. Kensler, T. W., A. K. Verma, R. K. Boutwell and G. C. Mueller. 1978. Effects of retinoic acid and juvenile hormone on the induction of ornithine decarboxylase activity by 12-O-tetradecanoylphorbol-13-acetate. Cancer Res. 38:2896-2899.

7. Wertz, P. W., T. W. Kensler, G. C. Mueller, A. K. Verma, and R. K. Boutwell. 1979. 5,6-Epoxyretinoic acid opposes the effect of 12-O-tetradecanoylphorbol-13-acetate in bovine lymphocytes. Nature (Lond.). 277:227-29.

8. Nordlind, K., and J. Thyberg. 1983. In vitro effects of 13-cis-retinoic acid (Ro 4-3780) and etretinate (Ro 10-9359) on DNA synthesis and fine structure of guinea pig and human lymphoid cells. Int. Arch. Allergy Appl. Immunol. 71:363367.
9. Eremin, O., J. Ashby, and J. Rhodes. 1984. Inhibition of antibody-dependent cellular cytotoxicity by retinoic acid. Int. Arch. Allergy Appl. Immunol. 75:2-7.

10. Blalock, J. E., and G. E. Gifford. 1976. Comparison of the suppression of interferon production and inhibition of its action by vitamin $A$ and related compounds (39532). Proc. Soc. Exp. Biol. Med. 153:298-300.

11. Hughes, T. K., J. K. Russell, and J. E. Blalock. 1986. Induction of interferon transformed cells: inhibition by retinoic acid. Biochem. Biophys. Res. Commun. 138:47-53.

12. Abb, J., H. Abb, and F. Deinhardt. 1982. Effect of retinoic acid on the spontaneous and interferon-induced activity of human natural killer cells. Int. J. Cancer. 30:307-310.

13. Abb, J., H. Abb, and F. Deinhart. 1982. Retinoic acid suppression of human leukocyte interferon production. Immunopharmacology. 4:303-310.

14. Rhodes, J., and P. Stokes. 1982. Interferon produced changes in the monocyte membrane: inhibition by retinol and retinoic acid. Immunology. 45:531536.

15. Uhr, J. W., G. Weissmann, and L. Thomas. 1963. Acute hypervitaminosis A in guinea pigs. II. Effects on delayed-type hypersensitivity. Proc. Exp. Biol. Med. 112:287-291.

16. Dresser, D. W. 1968. Adjuvanticity of vitamin A. Nature (Lond.). 217:527-528.

17. Spitznagel, J. K., and A. C. Allison. 1970. Mode of action of adjuvants: retinoid and other lysosome-labilizing agents as adjuvants. J. Immunol. 104:119127.

18. Dennert, G., and R. Lotan. 1978. Effects of retinoic acid on the immune system: stimulation of T killer cell induction. Eur. J. Immunol. 8:23-29.

19. Barnett, J. B. 1983. Immunomodulating effects of 13-cis-retinoic acid on the IgG and IgM response of BALB/c mice. Int. Arch. Allergy Appl. Immunol. 72:227-233.

20. Sidell, N., P. Rieber, and S. H. Golub. 1984. Immunological aspects of retinoids in humans. I. Analysis of retinoic acid enhancement of thymocyte responses to PHA. Cell. Immunol. 87:118-125.

21. Otani, S., I. Matsui-Yuasa, Y. Mimura, and S. Morisawa. 1986. Potentiation by retinoic acid of ornithine decarboxylase induction by phytohemagglutinin or phorbol 12-myristate-13-acetate in guinea pig lymphocytes. J. Biochem. 99:1789-1797.

22. Sidell, N., and F. Ramsdell. 1988. Retinoic acid upregulates interleukin-2receptor on activated human thymocytes. Cell. Immunol. 115:299-309.

23. Brinckerhoff, C. E., J. W. Coffey, and A. C. Sullivan. 1983. Inflammation and collagenase production in rats with adjuvant arthritis reduced with 13-cis-retinoic acid. Science (Wash. DC). 221:756-758.

24. Trentham, D. E., and C. E. Brinckerhoff. 1982. Augmentation of collagen arthritis by synthetic analogues of retinoic acid. J. Immunol. 129:2668-2672.

25. Massacesi, L., A. L. Abbamondi, C. Giorgi, F. Sarlo, F. Lolli, and L. Amaducci. 1987. Suppression of experimental allergic encephalomyelitis by retinoic acid. J. Neurol. Sci. 80:55-64.

26. Sedgwick, J., S. Brostoff, and D. W. Mason. 1987. Experimental allergic encephalomyelitis in the absence of a classical delayed-type hypersensitivity reaction: severe paralytic disease correlates with the presence of $I L-2$ receptor positive cells infiltrating the CNS. J. Exp. Med. 165:1058-1075.

27. Alvord, E. C., M. W. Kies, and A. J. Suckling. 1984. Experimental Allergic Encephalomyelitis: a Useful Model for Multiple Sclerosis. Alan R. Liss, Inc., New York.

28. Paterson, P. Y. 1960. Transfer of allergic encephalomyelitis in rats by means of lymph node cells. J. Exp. Med. 111:119-135.

29. Paterson, P. Y. 1976. Experimental autoimmune (allergic) encephalomyelitis: induction, pathogenesis, and suppression. In Textbook of Immunopathology. 2nd Edition. P. A. Miescher and H. J. Mueller-Eberhard, editors. Grune \& Stratton, Inc. New York. 179.

30. Ortiz-Ortiz, L., and W. O. Weigle. 1976. Cellular events in the induction of experimental allergic encephalomyelitis in rats. J. Exp. Med. 144:604-616.

31. Pettinelli, C. B., and D. E. McFarlin. 1981. Adoptive transfer of experimental allergic encephalomyelitis in $\mathrm{SJL} / \mathrm{J}$ mice after in vitro activation of lymph node cells by myelin basic protein: requirement for $\mathrm{Lyt}^{1+2-}$ lymphocytes. J. Immunol. 127:1420-1423.

32. Traugott, U. 1989. Detailed analysis of early immunopathologic events during lesion formation in acute experimental autoimmune ancephalomyelitis. Cell. Immunol. 119:114-129.

33. Richert, J. R., B. F. Driscoll, M. W. Kies, and E. C. Alvord. 1981. Experimental allergic encephalomyelitis: activation of myelin basic protein-sensitized spleen cells by specific antigen in culture. Cell. Immunol. 59:42-53.

34. Ben Nun, A., and Z. Lando. 1983. Detection of autoimmune cells proliferating to myelin basic protein and selection of $\mathrm{T}$-cell lines that mediate experimental allergic encefalomyelitis (EAE) in mice. J. Immunol. 130:1205-1213.

35. Norton, W. T., and S. E. Poduslo. 1973. Myelination in rat brain: method of myelin isolation. J. Neurochem. 21:749-758.

36. Deibler, G. E., R. E. Martenson, and M. W. Kies. 1972. Large scale 
preparation of myelin basic protein, from central nervous tissue of several mammalian species. Prep. Biochem. 2:139-165.

37. Smith, M. E., F. P. Somera, R. Saldivar, L. Massacesi, and J. Trotter. 1984. DNA changes in spinal cord of rats with experimental allergic encephalomyelitis. J. Neurochem. 43:1635-1641.

38. Kappler, J., and P. Marrack. 1986. Lymphokines. In Handbook of Experimental Immunology. Volume II. D. M. Weir, editor. Blackwell Scientific Publications, Ltd., Oxford, UK.

39. Gillis, S., W. Ferm, W. Ou, and K. A. Smith. 1978. T cell growth factor: parameters of production and a quantitative microassay for activity. J. Immunol. 120:2027-2032.

40. De Lean, A., P. J. Munson, and B. Rodbard. 1978. Simultaneous analysis of families of sigmoidal curves: applications to bioassay, radioligand assay, and physiological dose response curves. Am. J. Physiol. 235:E97-E102.

41. Panitch, H. S., and D. McFarlin. 1977. Experimental allergic encephalomyelitis: enhancement of cell-mediated transfer by concanavalin A. J. Immunol. 119:1134-1137.

42. Vane, F. M., J. K. Stoltemborg, and C. Y. L. Buggé. 1982. Determination of 13-cis-retinoic acid in human blood by reversed phase high performance liquid chromathography. J. Chromatogr. 227:471-484.

43. McCormick, A. M., J. L. Napoli, and H. F. DeLuca. 1978. High pressure liquid chromatographic resolution of vitamin A compounds. Anal. Biochem. 86:25-33.

44. Dougherty, T. F, and A. White 1945. Functional alterations in lymphoid tissue induced by adrenal cortical secretion. Am. J. Anat. 77:81-115.

45. Dougherty, T. F., and A. White. 1947. Evaluations of alterations produced in lymphoid tissue by pituitary adrenal cortical secretion. J. Lab. Clin. Med. 32:584-605.

46. MacPhee, I. A. M., F. A. Antoni, and D. W. Mason. 1989. Spontaneous recovery of rats from experimental allergic encephalomyelitis is dependent on regulation of the immune system by endogenous adrenal corticosteroids. $J$. Exp. Med. 169:431-442.

47. Kessler, J. F., F. L. Meyskens, N. Levine, P. J. Lynch, and S. E. Jones. 1983. Treatment of cutaneous T-cell lymphoma (mycosis fungoides) with 13-cisretinoic acid. Lancet. i:1345-1347.

48. Peck, G. L., T. G. Olsen, F. W. Yode, J. S. Strauss, D. T. Downing, M. Pandya, D. Butkus, and J. A. Buttandier. 1979. Prolonged remission of cystic and conglobate acne with 13-cis-retinoic acid. N. Engl. J. Med. 300:329-333.

49. Kerr, G. I., M. E. Lippmann, J. Jenkins, and C. Y. Myers. 1982. Pharmacology of 13-cis-retinoic acid in humans. Cancer Res. 42:2069-2073.

50. Clamon, G., G. G. Chabot, F. Valeriote, E. Davilla, C. Vogel, E. Gorowski, and R. Birch. 1985. Phase I study and pharmacokinetics of weekly highdose of 13-cis-retinoic acid. Cancer Res. 45:1874-1878.

51. Levine, S., R. Strebel, E. J. Wenk, and P. J. Harman. 1962. Suppression of experimental allergic encephalomyelitis by stress. Proc. Soc. Exp. Biol. Med. 109:294-298.

52. Bolton, B., J. F. Borel, M. L. Cuzner, A. N. Davison, and A. M. Turner.
1982. Immunosuppression by cyclosporine A of experimental allergic encephalomyelitys. J. Neurol. Sci. 56:147-153.

53. Levine, S., and R. Sowinski. 1980. Therapy of allergic encephalomyelitis in rats after onset of paralysis. In The Suppression of Experimental Allergic Encephalomyelitis and Multiple Sclerosis, A. N. Davison and M. L. Cuzner, editors. Academic Press, Ltd., London. 199-209.

54. Bedford, P. A., and S. C. Knight. 1989. The effect of retinoids on dendritic cell function. Clin. Exp. Immunol. 75:481-486.

55. Sporn, M. B., A. B. Roberts, L. M. Wakefield, and B. deCrombrugghe. 1987. Some recent advances in the chemistry and biology of transforming growth factor-B. J. Cell Biol. 105:1039-1045.

56. Glick, A. B., K. C. Flanders, D. Danielpour, S. H. Yuspa, and M. B. Sporn. 1989. Retinoic acid induces transforming growth factor $\beta_{2}$ in cultured keratinocytes and mouse epidermis. Cell Regul. 1:87-97.

57. Bollag, W., and F. Ott. 1989. Treatment of lichen planus with temarotene. Lancet. ii:974.

58. Chytil, F., and D. E. Yong. 1979. Cellular retinol- and retinoic acid binding proteins in vitamin A action. Fed. Proc. 38:2510-2514.

59. Giguerre, V., E. S. Ong, P. Segui, and R. M. Evans. 1987. Identification of a receptor for the morphogen retinoic acid. Nature (Lond.). 330:624-629.

60. Kastner, P. H., A. Krust, C. Mendelsohn, J. M. Garnier, A. Zelent, P. Leroy, A. Staub, and P. Chambon. 1990. Murine isoforms of retinoic acid receptor gamma with specific pattern of expression. Proc. Natl. Acad. Sci. USA. 87:2700-2704.

61. Mangelsdorf, D. J., E. S. Ong, J. A. Dick, and R. A. Evans. 1990. Nuclear receptor that identifies a novel retinoic acid response pathway. Nature (Lond.) 345:224-229.

62. Hashimoto, Y., H. Kagechika, and K. Shudo. 1990. Expression of retinoic acid receptor genes and the ligand binding selectivity of retinoic acid receptors (RAR's). Biochem. Biophys. Res. Commun. 166:1300-1307.

63. Blomhoff, R., M. H. Green, T. Berg, and K. R. Norum. 1990. Transport and storage of vitamin A. Science (Wash. DC). 250:339-404.

64. de The, H., A. Marchio, P. Tiollais, and A. Dejean. 1989. Differentia expression and ligand regulation of the retinoic acid receptor alfa and beta genes. EMBO (Eur. Mol. Biol. Organ.) J. 8:429-433.

65. Vasios, G. V., J. D. Gold, M. Petkovitch, P. Chambon, and L. J. Gudas 1989. A retinoic acid responsive element is present in the $5^{\prime}$ flanking region of the laminin B1 gene. Proc. Natl. Acad. Sci. USA. 86:9099-9103.

66. Rees, J. L., A. K. Daly, and C. P. F. Redfern. 1989. Differential expression of the alfa and beta retinoic acid receptors in tissues of the rat. Biochem. J. 259:917-919.

67. Evans, R. M. 1988. The steroid and thyroid hormone receptor superfamily. Science (Wash. DC). 240:889-895.

68. Larsen, P. D., and L. J. DeLallo. 1987. Cerebrospinal fluid transthyretin in multiple sclerosis. Neurology. 37:1262-1263.

69. Kalin, J. R., M. G. Wells, and D. L. Hill. 1982. Disposition of 13-cis retinoic acid and $N$-(hydroxyethyl)retinamide in mice after oral doses. Drug Metab. Dispos. 10:391-398. 